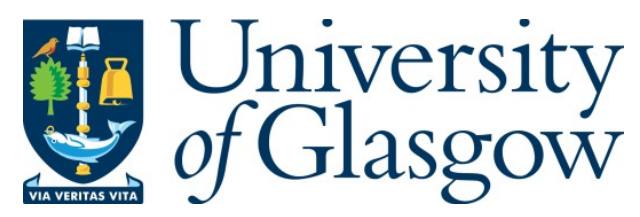

Moore, I. and Ceriotti, M. (2021) Solar sails for perturbation relief: application to asteroids. Advances in Space Research, 67(9), pp. 3027-3044.

(doi: 10.1016/j.asr.2020.08.014)

This is the Author Accepted Manuscript.

There may be differences between this version and the published version. You are advised to consult the publisher's version if you wish to cite from it.

https://eprints.gla.ac.uk/221942/

Deposited on: 10 August 2020

Enlighten - Research publications by members of the University of Glasgow http://eprints.gla.ac.uk 


\title{
Solar Sails for Perturbation Relief: Application to Asteroids
}

\author{
Iain MOORE ${ }^{\mathrm{a}, *}$, Matteo CERIOTTI ${ }^{\mathrm{a}}$ \\ ${ }^{a}$ James Watt School of Engineering, University of Glasgow, Glasgow, G12 8QQ, Scotland
}

\begin{abstract}
A major cause of spacecraft orbital variation comes from natural perturbations, which, in close proximity of a body, are dominated by its non-spherical nature. For small bodies, such as asteroids, these effects can be considerable, given their uneven (and uncertain) mass distribution. Solar sail technology is proposed to reduce or eliminate the net secular effects of the irregular gravity field on the orbit. Initially, a sensitivity analysis will be carried out on the system which will show high sensitivity to changes in initial conditions. This presents a challenge for optimisation methods which require an initial guess of the solution. As such, the Genetic Algorithm (GA) is proposed as the preferred optimisation method as this requires no initial guess from the user. A multi-objective optimisation is performed which aims to achieve a periodic orbit whilst also minimising the effort required by the sail to do so. Given the system sensitivity, the control law for one orbit is not necessarily applicable for any subsequent orbit. Therefore, a new method of updating the control law for subsequent orbits is presented, based on linearisation and use of a Control Transition Matrix (CTM). The techniques will later find application in a multiple asteroid rendezvous mission with a solar sail as the primary propulsion system.

Keywords: Asteroids, Perturbations, Solar Sail, Optimal Control, Spherical Harmonics
\end{abstract}

${ }^{*}$ Corresponding author, i.moore.3@research.gla.ac.uk 


\section{Nomenclature}

$a$

$a_{c}$

$b$

C

$d$

$e$

$h$

$i$

$I_{z z}$

$J$

$M$

$m$

$\mathbf{n}$

$\hat{\mathbf{n}}$

$P$

$\mathbf{r}$

$r_{0} \quad$ Normalising radius

$r_{0, \text { points }}$ Radius of sphere containing initial points

$\mathbf{r}_{\text {sun }} \quad$ Sun position vector

Semi-major axis

Characteristic acceleration

Dimension of spacecraft/sail

Gravity field harmonic coefficients

Dimension of spacecraft/sail

Eccentricity

Dimension of spacecraft/sail

Inclination

Moment of inertia around principal $z$-axis

Objective function

Mass of asteroid

Mass of spacecraft/sail

Sail normal vector

Unit vector of sail normal vector

Associated Legendre functions

Position vector 
$\hat{\mathbf{r}}_{\text {sun }}$ Unit vector of Sun position

s Cartesian state vector

$S \quad$ Gravity field harmonic coefficients

$t \quad$ Time

$U \quad$ Gravity field potential function

u Control vector

$X_{e} \quad$ Ellipsoid dimension

$Y_{e} \quad$ Ellipsoid dimension

$Z_{e} \quad$ Ellipsoid dimension

$\alpha \quad$ Sail cone angle

$\gamma \quad$ Angle between two sail attitudes

$\dot{\gamma} \quad$ Magnitude of rigid-body angular velocity of sail

$\Delta \dot{\gamma} \quad$ Total changes in $\dot{\gamma}$ over one orbit (Sail "effort" value)

$\delta \quad$ Sail clock angle

$\epsilon \quad$ Error in state

$\theta \quad$ True anomaly

$\lambda \quad$ Longitude

$\mu \quad$ Gravitational parameter

$\sigma \quad$ Density

$\tau \quad$ Torque of rigid-body sailcraft 


$\begin{array}{ll}\Phi_{C} & \text { Control transition matrix } \\ \Phi_{S} & \text { State transition matrix } \\ \phi & \text { Latitude } \\ \Omega & \text { Right ascension of ascending node }\end{array}$

Superscripts

T Transpose

Subscripts

$c$

Denotes relation to cube

$l \quad$ Degree of harmonic expansion

$m \quad$ Order of harmonic expansion

new Denotes relation to new orbit obtained by CTM method

nominal Denotes relation to nominal orbit

$p \quad$ Denotes relation to plate

$0 \quad$ Initial point

$f \quad$ Final point

\section{Introduction}

Asteroids, and in particular near-Earth asteroids (NEAs), pose both a threat and an opportunity to life on Earth: a threat in terms of possible Earth impact, and an opportunity to explore what are often pristine relics of the early solar system. In more recent years, NEAs have also become the focus of a new breed of space-entrepreneur: the asteroid miner.

Given the limitations of Earth-based observations, it is desirable to conduct 
in-situ analysis of these bodies. There have been several successful asteroid missions and, at the time of writing, there are two active missions: Hayabusa 2 and OSIRIS-REx. JAXA's Hayabusa 2 mission (Sarli and Tsuda, 2017) has so far brought many successes, not least in the deployment of the MASCOT landers (Ho et al. 2017). This mission will perform a sample extraction to be returned to the Earth for analysis and follows the earlier Hayabusa mission (Yoshikawa et al., 2006) which also, successfully, returned a sample of asteroid Itokawa to the Earth in 2010. NASA is also carrying out a sample return mission with OSIRIS-REx (Hesar et al., 2016), which will return samples from asteroid Bennu to the Earth. NASA will launch the first solar sail mission to an asteroid when NEA Scout (McNutt et al. 2014) begins its 2 year journey to flyby asteroid 1991VG atop the new Space Launch System (SLS). There have also been other flyby and rendezvous proposals made in the literature (Bando and Yamakawa, 2011; Hughes and McInnes, 2004, Zeng et al., 2014), which promise to harness the unique ability of solar sail technology to provide high energy missions. In their Gossamer roadmap series of papers, Dachwald et al. (2014); Grundmann et al. (2015, 2019) continue the two decades of work from DLR and ESA in developing the path to solar sailing missions. One of the recent stages of this roadmap shows the unique flexibility of the technology in making possible a single mission to multiple NEAs (Peloni et al., 2016).

A key phase of the mission to an asteroid will be the operation of the spacecraft in the near-asteroid space. In this region, the effects of the non-spherical nature of the body become very important. For highly irregular bodies, these perturbations can lead to orbital escape (Scheeres, 1999). Therefore, it is beneficial to have a method of alleviating these effects on the spacecraft.

Macdonald and McInnes (2005) propose the blending of optimal control laws for station-keeping manoeuvres. Application is made to the Earth-centred "GeoSail" mission, where the orbit lies in the ecliptic plane. In order to maintain a Sun-pointing periapsis, and so the apoapsis in the Earth's magnetotail, the sail orientation is chosen such that the accelerations experienced are able to countenance the secular variation of the argument of periapsis. 
Biggs and McInnes (2009) present the application of Time-Delayed Feedback Control (TDFC) as a method of bounding the orbit of a spacecraft around a highly eccentric ellipsoid; in this case applied to asteroid 433 Eros. Rather than depending on some reference trajectory, this method utilises the state known one period previous to the current state as the reference. Such a method allows for an adaptive approach to the problem; very important where the gravity field is not known a priori, which is most often the case for an asteroid mission.

Oliveira and Prado (2014) present their work in station keeping manoeuvres using a solar sail to combat perturbations from numerous sources. This work utilises PID control of the solar sail to counteract the accelerations experienced by the spacecraft due to perturbations. The method finds success in addressing the accelerations experienced, and shows the versatility of the solar sail in mission applications.

In a series of works, Farrés et al. propose using the sail in order to offset undesirable effects from the non-linear dynamics of multi-body systems. Farrés and Jorba (2012); Farrés and Jorba (2016) propose to use the sail to stabilise a spacecraft's orbit around an unstable equilibrium point in the Circular Restricted 3 Body Problem (CR3BP). The objective is to allow the sail to escape along the unstable manifold before changing the sail orientation such that the stable manifold is brought into the path of the sail, thus allowing the spacecraft to remain bound in orbit around the equilibrium point. Later, this strategy was applied to a halo orbit in the CR3BP (Farrés and Jorba, 2014). Farrés and Jorba (2011) then apply the same method to the Elliptical Restricted 3 Body Problem (ER3BP). Farres and Ceriotti (2012) propose strategies of stabilising vertical Lyapunov orbits in the CR3BP using both LQR control and Floquet Modes.

The current work aims to use the solar sail to counteract the effect of the perturbations from a non-spherical body on a spacecraft. A multi-objective optimisation will be performed using a Genetic Algorithm (GA) to obtain a control law which converges to a periodic orbit while also minimising the effort required by the sail to do so. As the dynamics are very sensitive, in most cases, 
any control law will not be valid for any small displacement from the GA nominal orbit after one or more revolutions. Therefore, a method will be presented for updating the control law for subsequent orbits in the neighbourhood of the nominal one. This method allows the sail to converge to a periodic orbits, up to a certain inclination. The limiting effect of orbital inclination will also be analysed.

The paper is organised as follows. Section 2 discusses the different shape models available for asteroids and makes the case for the selected model. Section 3 introduces the dynamics of the problem before a sensitivity analysis of the asteroid system is performed in Section 4. The methodology is described in Section 5. This will include an analysis of the multi-objective optimisation and a method proposed for updating the control law for orbits subsequent to the nominal. Results from each of the described methods will be presented in Section 6 along with an analysis on the limiting effect of the orbit inclination on the success of the method of updating the control law in Section 6.4. Finally, Section 7 introduces a smoother control law by application of cubic splines interpolation to provide a real world applicable control law. The torque required to perform these manoeuvres are then presented and compared with similar results from the literature for realistic sails.

\section{Asteroid Shape Model Selection}

Asteroids are of such a small mass that their gravitational force is not strong enough to compact their constituent materials into the spherical shape which we see for larger bodies (Cox and Cohen, 2017). As such, they tend to have very irregular shapes, which give rise to irregular gravity fields. This poses a problem in choosing a model accurate enough to be able to predict the spacecraft motion around the asteroid with sufficient precision for navigation.

The literature has shown the use of many different gravity field models, as researchers seek the balance between accuracy of results and efficiency of computation. Several authors (Scheeres, 2011; Hu and Jekeli, 2015, Sebera et al. 
2016 ) discuss the use of harmonic expansion methods; spherical, spheroidal and ellipsoidal. The harmonic expansions are limited by the Brillouin surface. This surface represents the boundary of convergence for these methods. External to the surface, the method will converge. However, the standard methods do not guarantee convergence either close to, or internal to, the surface. Takahashi and Scheeres (Takahashi and Scheeres, 2014) discuss the interior Brillouin surface where the gravity field converges within the Brillouin surface. This is important in modelling the dynamics of a spacecraft very close to the asteroid surface, though only possible when some knowledge of the asteroid shape is available.

Further more authors have utilised the ellipsoid as a reference shape for the asteroid and have modelled this using the tri-axial ellipsoid calculated using elliptical integrals (Guibout and Scheeres, 2003; Scheeres, 1993, 1994, 2011).

Rossi (1999) describes the use of discrete point masses which define an irregularly shaped body. The problem then becomes an $n$-body problem, where the potential of each point mass is summed to give the total potential of the body.

Other method such as the use of a dumb-bell model (Gozdziewski, 1998) and a cube model (Venditti and Prado, 2015) have also been proposed.

The most accurate method of modelling asteroids is through use of shape models which have been obtained via light curve data, radar, or in-situ analysis. These models can then be represented using polyhedron modelling. This allows for very detailed models of the asteroid where the true, irregular, shape of the body can be accurately represented. This method is described and applied by numerous authors (Scheeres, 2004, 2012, Mota and Rocco, 2019, Rocco, 2019, Werner, 1994; Scheeres et al., 1996, Werner and Scheeres, 1997; Rossi, 1999, Viale et al., 2019) and the work allows a much higher fidelity model to be produced than is possible with any other method. However, this accuracy comes at a computational cost, as the calculation of the potential required summation over the face and each edge of every polygon.

The choice of which model to use is highly dependent on both the distance of the spacecraft to the asteroid and also the precision of the gravity field needed for trajectory design and spacecraft navigation. When the spacecraft is close to 
the surface, and/or interacting with the surface, then the highly detailed and accurate polyhedron models are required. If the spacecraft is more distant from the surface then the computationally intensive polyhedron models are no longer required, as the effect of the complex asteroid shape on the gravitational field quickly dissipate.

To understand the effects of the shape of a body on the gravitational field, Fig. 1 shows the contours for the magnitude of the gravitational acceleration for four asteroids: Castalia, Geographos, Itokawa and Mithra, using the polyhedron shape models. These are examples of elongated, or highly irregular, bodies. The contours, plotted in the $x-y$ plane for each asteroid, show the shape of the gravity field. It is clear that the shape of the field quickly becomes more regular as the point mass moves radially outward from the surface of the body.

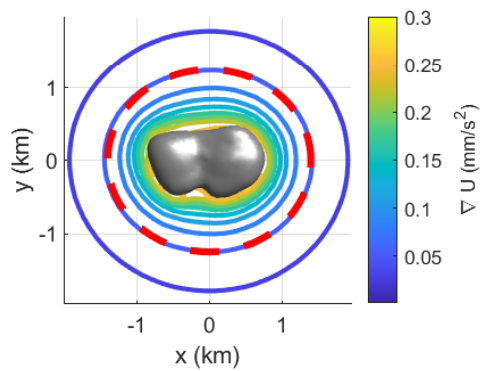

(a) Castalia

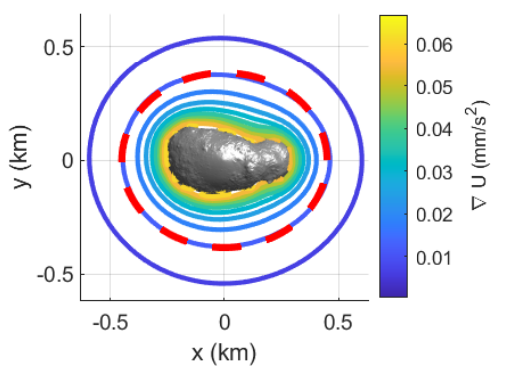

(c) Itokawa

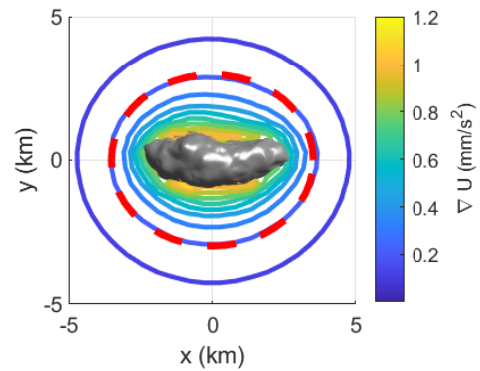

(b) Geographos

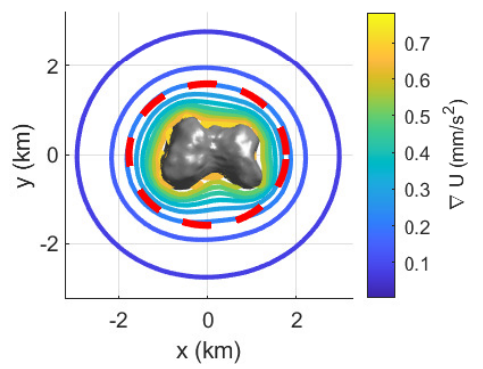

(d) Mithra

Fig. 1: Contour plots of magnitude of the gravitational acceleration in the $x-y$ plane for asteroids Castalia, Geographos, Itokawa and Mithra. The red line shows the elliptical approximation of the given contour line. 
Given the increasing regularity of the shape of the gravity field as the point mass moves away from the body, it becomes wholly reasonable to assume a less computationally expensive method where the simulated work remains at these distances from the surface. The red dashed line of Fig. 1 1 shows an ellipse which is fitted to a particular contour line. In order to understand the accuracy of approximating the contour as an ellipse, the Mean Squared Error (MSE) of the ellipse relative to the contour line is calculated for each asteroid and presented in Table. 1 .

Table 1: Mean squared error of elliptical approximation of gravity field contour lines.

\begin{tabular}{|c|c|}
\hline & MSE $\left(\mathrm{km}^{2}\right)$ \\
\hline Castalia & $3.03 \times 10^{-4}$ \\
\hline Geographos & $7.20 \times 10^{-2}$ \\
\hline Itokawa & $1.03 \times 10^{-4}$ \\
\hline Mithra & $7.67 \times 10^{-2}$ \\
\hline \hline
\end{tabular}

It is clear from the small values of MSE that an elliptical approximation is indeed very accurate, and so this work will use the ellipsoid to model the irregular asteroid

\section{Dynamics}

This work uses two-body dynamics where the gravitational potential field of one massive body (the asteroid) acts on a negligible-mass spacecraft. This allows focus on gravitational perturbations from the irregular gravity field of that body only, and no third-body effects. The inertial reference frame is centred on the asteroid centre of mass with the $x$-axis in the direction of the first point of Aries, the $z$-axis pointing along the axis of rotation and the $y$ axis completing the right-handed set. Pravec et al. (2002) state that, for asteroids of diameter smaller than $10 \mathrm{~km}$, there are significant populations of both slow and fast rotators, which can have rotational periods from longer than one day to as 
short as 2 hours. As such, the asteroid is considered to have a rotational period of 5 hours, which is within this range. At the origin of time, the asteroid longest dimension is aligned with the $x$-axis of the inertial frame.

The two-body acceleration for a spacecraft in this system is given by:

$$
\ddot{\mathbf{r}}=\nabla U
$$

where $\ddot{\mathbf{r}}$ is the acceleration of the spacecraft, and $\nabla U$ is the gradient of the gravitational potential function of the non-spherical body, modelled using spherical harmonics. This potential function $U$ is given by Scheeres (2011):

$$
\begin{aligned}
U(r, \phi, \lambda) & =\frac{\mu}{r} \sum_{l=0}^{\infty} \sum_{m=0}^{l}\left(\frac{r_{0}}{r}\right)^{l} P_{l m}[\sin \phi] \times \ldots \\
& \ldots \times\left\{C_{l m} \cos m \lambda+S_{l m} \sin m \lambda\right\} \\
P_{l m}[\sin \phi] & =\cos ^{m} \phi \sum_{i=0}^{i n t[(l-m) / 2]} T_{l m i} \sin ^{l-m-2 i} \phi \\
T_{l m i} & =\frac{(-1)^{i}(2 l-2 i) !}{2^{l} i !(l-i) !(l-m-2 i)}
\end{aligned}
$$

where $\mu$ is the asteroid gravitational parameter, $r$ is the magnitude of the spacecraft position relative to the asteroid, $l$ is the degree of the harmonic expansion, $m$ is the order of the harmonic expansion, $r_{0}$ is the mean radius of the asteroid, $P$ is the associated Legendre function, $\phi$ is the latitude of the spacecraft position in an asteroid centred reference frame, and $C_{l m}, S_{l m}$ are the harmonic coefficients of the model. The coefficients for an ellipsoid, given by Scheeres (2011), will be applied: 


$$
\begin{aligned}
C_{20} & =\frac{1}{5 r_{0}^{2}}\left(Z_{e}^{2}-\frac{X_{e}^{2}+Y_{e}^{2}}{2}\right) \\
C_{22} & =\frac{1}{20 r_{0}^{2}}\left(X_{e}^{2}-Y_{e}^{2}\right) \\
C_{40} & =\frac{15}{7}\left(C_{20}^{2}+2 C_{22}^{2}\right) \\
C_{42} & =\frac{5}{7} C_{20} C_{22} \\
C_{44} & =\frac{5}{28} C_{22}^{2}
\end{aligned}
$$

where $r_{0}$ is the normalising radius of the body, which in the case of the ellipsoid is taken as the largest dimension, and $X_{e}, Y_{e}, Z_{e}$ are the ellipsoid dimensions. Fig. 2 shows the dimensions of the ellipsoid.

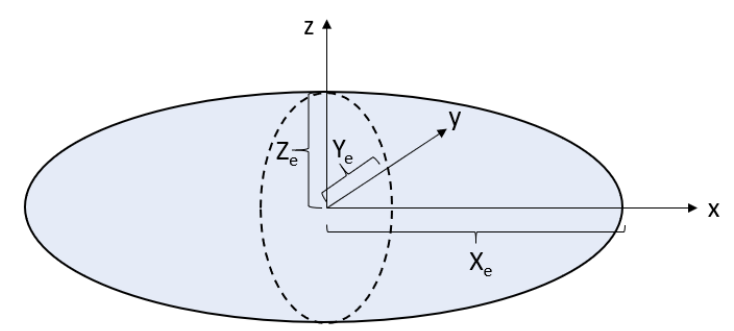

Fig. 2: Dimensions of ellipsoid, as presented in Scheeres (2011)

In order to establish the mass of the body, we assume an average asteroid density of $\sigma=3.5 \mathrm{~g} / \mathrm{cm}^{3}$ (Scheeres, 2011). The mass is then given by:

$$
M=\frac{4 \pi \sigma X_{e} Y_{e} Z_{e}}{3}
$$

Finally, the acceleration due to the solar sail (McInnes, 1999) is added:

$$
\ddot{\mathbf{r}}=\nabla U+a_{c}\left(\hat{\mathbf{r}}_{\text {sun }} \cdot \hat{\mathbf{n}}\right)^{2} \hat{\mathbf{n}}
$$

where $\hat{\mathbf{r}}_{\text {sun }}$ is the unit vector of the Sun-line, $\hat{\mathbf{n}}$ is the sail normal unit vector and $a_{c}$ is the sail characteristic acceleration. In this work, an ideal, perfectly reflecting sail is used. The ideal sail neglects the effects of imperfect reflection as well as those from a billowing sail. These effects can both reduce the effective 
performance of the sail as well as result in a thrust vector which is not aligned with the sail normal, leading to additional challenges in control.

The assumption that the NEA is in a circular orbit around the Sun at $1 \mathrm{AU}$ is made, and the time considered for operations around the asteroid are short enough that any change in position of the Sun can be neglected. Therefore, $\hat{\mathbf{r}}_{\text {sun }}=[1,0,0]$. This work will not consider the effect of eclipses, where the sail passes behind the asteroid with respect to the Sun, and so where there would be no effect from SRP.

The definition of the system dynamics is completed by establishing the sail normal vector (McInnes, 1999):

$$
\hat{\mathbf{n}}=[\cos (\alpha), \sin (\alpha) \cos (\delta), \sin (\alpha) \sin (\delta)]^{T}
$$

as a function of the control angles, sail cone $\alpha$ and clock angle $\delta$.

\section{System Sensitivity}

Optimisation methods, particularly local ones, usually require initialisation with an initial guess of the solution. This initial guess must be sufficiently close to the optimal solution for the optimisation method to converge to a solution. For highly sensitive systems, it becomes necessary for such an initial guess to be very close to the optimal solution in order for the optimisation method to converge. To establish a suitable method of optimisation, the orbit sensitivity to changes in initial condition is analysed here.

Fig. 3 shows this analysis for a range of four different orbits, with orbital elements shown in Table 2. Incremental changes are made in the Cartesian $x$, $y$ and $z$ initial positions and the final error in $x, y$ and $z$ positions after one full revolution, relative to the final position of the reference orbit, are recorded. This final error in each of the $x, y$ and $z$ co-ordinates are shown on the $y$-axis of Fig. 3 with the changes in initial position shown along the $x$-axis. Therefore, the final point error is the distance between the final point of the reference orbit, and the final point for the perturbed orbit. 


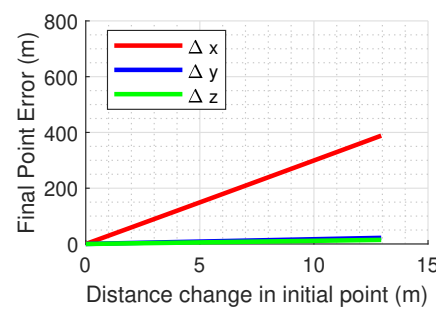

(a) Sensitivity analysis with orbital inclination of 0 degrees

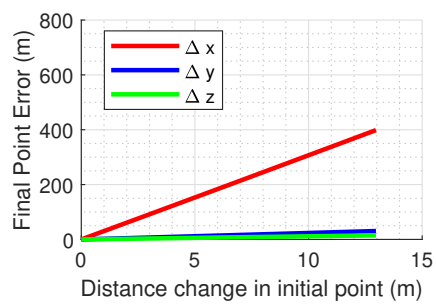

(c) Sensitivity analysis with orbital inclination of 30 degrees

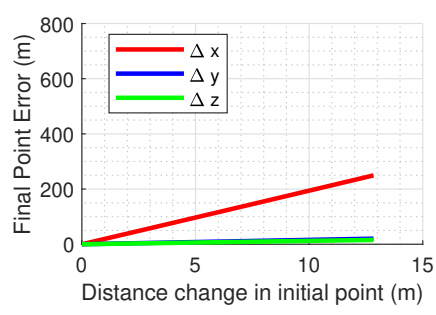

(b) Sensitivity analysis with orbital inclination of 15 degrees

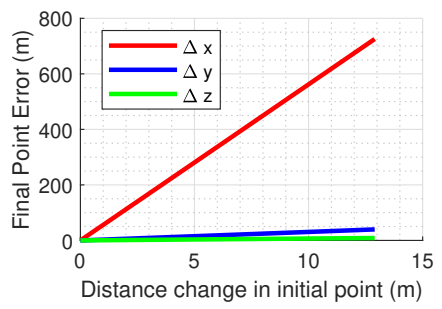

(d) Sensitivity analysis with orbital inclination of 60 degrees

Fig. 3: Sensitivity analysis for range of orbital inclinations.

Table 2: Principal orbital elements for four orbits tested in sensitivity analysis

\begin{tabular}{|l|c|c|c|}
\hline \hline & $a(\mathrm{~km})$ & $e$ & $i(\mathrm{deg})$ \\
\hline a) & 9.80 & 0.10 & 0 \\
\hline b) & 9.80 & 0.10 & 15 \\
\hline c) & 9.80 & 0.10 & 30 \\
\hline d) & 9.80 & 0.10 & 60 \\
\hline \hline
\end{tabular}

It is clear that the system is most sensitive to changes along the $x$-axis for all orbits, with the highest sensitivity seen in the highest inclination orbit (as the orbits' initial point is always at periapsis, changes in $x$-position impact mostly the semi-major axis). At an orbital inclination of $i=60^{\circ}$, for every metre change in initial condition along the $x$-axis, there is an associated error of $46 \mathrm{~m}$ in final position when compared to the final position of the original orbit.

Given this high sensitivity, it can be difficult for any optimisation method 
to converge to a solution to the optimisation problem, unless the initial guess is very close to the optimal solution. A wide variety of cases were tested using a Gauss pseudospectral method of optimal control (Rao et al., 2010). However, these failed to converge. As such, and without knowledge of a solution which would be sufficiently close to the optimal solution, it is desirable to use an optimisation method which does not require an initial guess from the user.

\section{Methods}

In this work, the objective is to design stable periodic orbits which can be controlled by a solar sail. To do this, a multi-objective optimisation will be performed by a Genetic Algorithm (GA) where the objectives are to minimise the error in initial and final state, so as to ensure periodicity, while also minimising the effort required by the sail to do so.

Solar sails possess high moments of inertia. As such, high rates of rotation would place excessive demands on the system power supply or would necessitate excessively heavy reaction wheels. As such, the second objective of minimising the effort allows the optimisation to find control laws which are feasible for near-term sails.

\subsection{Multi-Objective Optimisation with Genetic Algorithm}

A Genetic Algorithm (GA) provides a method for both constrained and unconstrained optimisation based on the principles of evolution. Having defined "individuals" that encode the solution vector, the algorithm combines pairs of "parents" to produce the next generation of "children". Over successive generations, the "population" will converge to the optimal solution.

The benefit of this algorithm, for the purposes of this work, is that it requires no initial guess from the user. The algorithm can be initialised with an initial population randomly spread in the search space. However, it is important to note that the algorithm is stochastic, and the convergence to the global optimum is not guaranteed; in fact, a solution provided from one initial population can 
be different from the optimal solution for another initial population. As such, it is necessary to run multiple instances, or "seeds", in order to obtain a true optimal solution.

From the full set of solutions to the multi-objective optimisation problem, there exists a subset of non-dominated solutions which, when plotted in the objective-space, produce what is referred to as the Pareto Front (Dirkx and Mooij, 2017). The GA provides both Pareto and non-Pareto solutions. In order to build a better Pareto front based on all solutions, all solutions from all seeds are aggregated and from these, the non-dominated solutions from this full set are selected to produce the Pareto front.

There exists then the question of how to select the appropriate solution from the multi-objective optimisation. Rocco et al. (2003) provide an overview of the many optimisation methods available and, along with Rocco et al. (2013), discuss the selection of solutions from the Pareto Front. It is possible to apply a weighting to each solution, but this must be carefully chosen so as not to bias a particular objective. The choice of weighting applied to each Pareto optimal solution, in itself, presents an optimisation problem, and it also fails to take advantage of the population based-nature of the Genetic Algorithm (Coverstone-Carroll et al., 2000).

In the optimisation of the asteroid orbit, two objectives are considered:

1. Reduce the error in the state-space between the initial and final point of an orbit, this is to ensure a periodic orbit.

2. Minimise the effort required by the sail to do so, in order to meet realworld steering constraints of a sail and the requirements this places in terms of power and weight.

Therefore, the objective functions for this work can be formulated as:

$$
\begin{aligned}
& J_{1}=\left\|\mathbf{s}\left(t_{f}\right)-\mathbf{s}\left(t_{0}\right)\right\| \\
& J_{2}=\int_{t_{0}}^{t_{f}} \ddot{\gamma} \mathrm{dt}
\end{aligned}
$$


where $\mathbf{s}$ is the state vector of the spacecraft, $t_{0}$ and $t_{f}$ are the initial and final times respectively and $\dot{\gamma}$ is the magnitude of the angular velocity of the sail (as a rigid body) as it changes attitude. By taking the integral of the angular acceleration $(\ddot{\gamma})$ of the sail over one orbital period, the cumulative change in angular velocity, $\Delta \dot{\gamma}$, is obtained. This value will be considered the total "control effort" required by the sail.

To reduce the time-continuous optimal control problem to a static problem, the control will be discretized into $N$ nodes, equally spaced in time, along the trajectory, and then interpolated to reconstruct a continuous control history. Initially, the control will be interpolated using a piece-wise-constant method. Later, a differentiable cubic spline interpolation will be presented as a viable control for a realistic sail. The optimisation solution vector will therefore be composed of the controls at each node, the final time of the orbit and the initial state of the orbit. As there are $N$ discrete nodes, and the control has two components $(\mathbf{u}=[\alpha, \delta])$, there will be $2 N$ controls. Bounds for the cone angle are set such that $\alpha \in[0, \pi / 2]$, the clock angle bounds are set such that $\delta \in[0,2 \pi]$. The bounds applied to the final time are set at $20 \%$ either side of the final time of the unperturbed orbit. For the initial state, the bounds are applied as a percentage of the magnitude of the reference initial state. In this work, there will be a $\pm 5 \%$ tolerance on each component of the Cartesian initial state. The control law obtained from this process will be the nominal control, $\mathbf{u}_{\text {nominal }}$.

\subsection{Updating the Control Law}

Given the system sensitivity, which was illustrated in Section 4 any small discrepancy between the initial and final state of an orbit could mean that the nominal control law, established by the optimisation method of Section 5.1 . cannot maintain periodicity for any subsequent orbit. As such, a method by which the control law can be updated using the available information (the error in initial and final states) is desirable. 


\subsubsection{Control Transition Matrix}

The errors in state at time $t$, due to variations at $t_{0}$, are given by (to a linear approximation):

$$
\delta \mathbf{s}(t)=\Phi_{S}\left(t, t_{0}\right) \delta \mathbf{s}\left(t_{0}\right)
$$

where $\Phi_{S}$ is the State Transition Matrix (STM) (Wiesel, 2010, Vallado, 2013). In this work, the interest lies in the effect that variations of control, rather than initial state, will have on the final state of the spacecraft. As such, this work now extends the linear theory of the STM to include these control variations. An equivalent matrix to the STM will be referred to as the Control Transition Matrix (CTM). Each entry of the CTM represents the variation in the final states for a unitary variation in each of the controls. The matrix size is therefore $6 \times 2 N$.

By replacing $\Phi_{S}$ with $\Phi_{C}$, the CTM:

$$
\delta \mathbf{s}\left(t_{f}\right)=\Phi_{C} \delta \mathbf{u}
$$

where $\delta \mathbf{u}$ are the deviations in control vector $\mathbf{u}_{\text {nominal }}$. The objective is to correct the nominal control law to account for errors in the initial state. The variation of the controls that produces a variation in $\delta \mathbf{s}\left(t_{f}\right)$, is (at least in a first order approximation):

$$
\delta \mathbf{u}=\Phi_{C}^{-1} \delta \mathbf{s}\left(t_{f}\right)
$$

Therefore, in order to remove an error in $\delta \mathbf{s}_{0}$ after one period, the aim is to achieve a final error of:

$$
\delta \mathbf{s}\left(t_{f}\right)=-\delta \mathbf{s}_{0}
$$

This assumes that the linearisation of the CTM is still valid for the perturbed orbit, allowing the perturbed orbit to be corrected towards the nominal orbit. As the CTM is not a square matrix, its inverse is not uniquely defined. The 
Moore-Penrose pseudo-inverse is used to obtain $\Phi_{C}^{-1}$, which results in a $2 N \times 6$ matrix. To update the control law, the nominal control is added to the control variation:

$$
\mathbf{u}=\mathbf{u}_{\text {nominal }}+\delta \mathbf{u}
$$

\subsubsection{Monte Carlo method to establish success rates}

Monte Carlo methods are a set of numerical methods based on repetitive random sampling to assess the success of a given system under uncertain initial conditions. For this work, a set of initial states, $\mathbf{s}_{i}$, is generated randomly, and used to test the success rates of the method described in Section 5.2.1. A sphere of radius $r_{0, \text { points }}$ will contain 500 normally distributed random points within 3 standard deviations of the mean. To generate these points, Ceriotti and Sanchez (2016) give:

$$
\mathbf{s}_{i}=\mathbf{s}_{\text {nominal }}+\left(r_{0, \text { points }} \frac{\text { rndNorm }}{1.96}\right) \mathbf{1}_{(6 \times 1)}
$$

where $r_{0, \text { points }}$ is the radius of sphere containing points, rndNorm is a randomly generated number with normal distribution, the 1.96 scaling factor guarantees that the randomly generated points are within 3 standard deviations of the mean, giving a $97.5 \%$ probability that the points will be contained in the sphere. The $\mathbf{1}_{(6 \times 1)}$ matrix is a $6 \times 1$ matrix of ones.

\subsubsection{Success Parameters}

With the method of updating the control law established, it is now useful to define success parameters for this method. Fig. 4 shows the initial state of the nominal orbit, $\mathbf{s}_{0, \text { nominal }}$, on a Poincaré section (Wiesel, 2010), where the black dot represents the point at which the section is pierced. The success of the method will consider a displaced orbit, $\mathbf{s}_{0}$, which will be propagated with both the nominal control law (red line) and the updated control law (blue line).

$\mathbf{s}_{f, \mathbf{u}_{\text {new }}}$ is the final state of the orbit which originates at $\mathbf{s}_{0}$ and utilises the new control law, $\mathbf{u}(t)$, and $\mathbf{s}_{f, \mathbf{u}_{\text {nominal }}}$ is the final state of an orbit which 


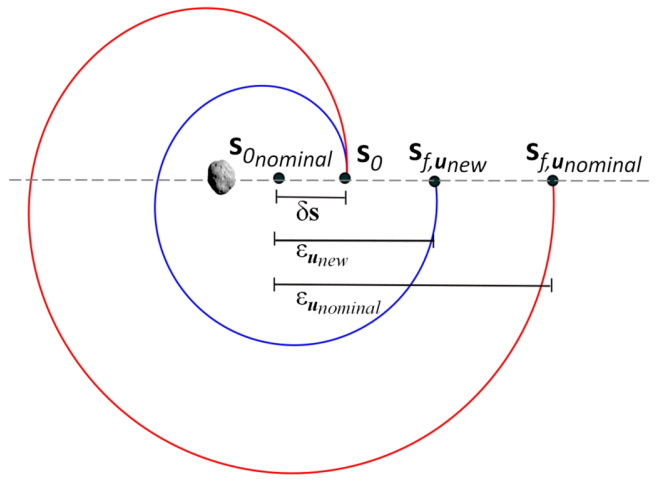

Fig. 4: Schematic showing the initial and final states of the nominal orbit, new orbit, and the error in the states and their final states if propagated with both the new control and the nominal control. The Poincaré section, which lies in the $x-z$ plane of the asteroid-centred reference frame, is represented by the grey dashed line. Each trajectory orbits once around the asteroid.

originates at $\mathbf{s}_{0}$ and is propagated using the nominal control law. $\delta \mathbf{s}$ is the error between the nominal orbit initial state and the initial state of the orbit originating at $\mathbf{s}_{0}, \epsilon_{\mathbf{u}_{\text {new }}}$ is the error between the nominal initial point and the final point of the orbit originating at $\mathbf{s}_{0}$ and propagated using the new control law and $\epsilon_{\mathbf{u}_{\text {nominal }}}$ is the error between the nominal initial state and the final state of the orbit originating at $\mathbf{s}_{0}$ propagated using the nominal control law.

The first success parameter is defined as $\epsilon_{\mathbf{u}_{\text {new }}}<|\delta \mathbf{s}|$, which implies that the updated control law $\mathbf{u}$ in Eq. 13 reduces the norm of the initial state error after one orbit. The second success parameter is defined as $\epsilon_{\mathbf{u}_{\text {new }}}<\epsilon_{\mathbf{u}_{\text {nominal }}}$. This success parameter shows that by integrating the updated control law, the final state is closer to the target final state than would have been achieved by simply using the nominal control law again.

\section{Results}

\subsection{Multi-Objective Optimisation with Genetic Algorithm}

In this section a target orbit with elements listed in Table 3 is sought by the GA multi-objective optimisation. The objectives are for a periodic orbit with a 
minimum amount of effort required by the sail.

Table 3: Principal orbital elements for the initial target orbit for the GA multi-objective optimisation.

\begin{tabular}{|c|c|c|}
\hline \hline$a(\mathrm{~km})$ & $e$ & $i(\mathrm{deg})$ \\
\hline 9.80 & 0.10 & 15.00 \\
\hline \hline
\end{tabular}

As discussed in section 5.1 it is necessary to initialise the GA with multiple seeds. In this work, 25 seeds are used to generate 25 initial populations and their solutions form the full solution set. The non-dominated solutions from this set form the Pareto front.

Fig. 5 shows the full solution set in black points with the Pareto Front shown in red.

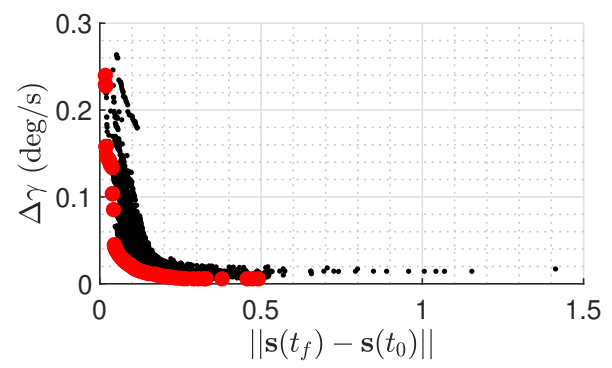

Fig. 5: Pareto front shown in red with full set of solutions in black.

It is clear that, for a minimum amount of effort from the sail, there will be a correspondingly high difference between the initial and the final state of the orbit. Similarly, a minimum difference in final and initial state will bring a higher effort value. Section 5.1 discussed the methods which are available in the literature for selection of the appropriate solution from the Pareto front. However, it should be noted that data from The Planetary Society's LightSail 2 mission shows rotation rates of $\dot{\gamma}=0.4 \mathrm{deg} / \mathrm{s} \rrbracket$. As such, a solution is sought

\footnotetext{
${ }^{1}$ https://www.planetary.org/explore/projects/lightsail-solar-sailing/lightsail-missioncontrol.html, cited March 27th, 2020
} 
which offers an effort value which is lower than $\Delta \dot{\gamma}=0.4 \mathrm{deg} / \mathrm{s}$ and which offers as close to a periodic solution as possible. As all of the solutions on the Pareto Front meet the effort condition, the solution which offers the minimum difference in final and initial states is chosen. The control law and trajectory for the chosen solution are shown in Fig. 6. The difference in initial and final states is reduced by allowing variation in initial state from the reference initial state as part of the optimisation. For this orbit the objective values were $\left\|\mathbf{s}\left(t_{f}\right)-\mathbf{s}\left(t_{0}\right)\right\|=0.017$ and $\Delta \dot{\gamma}=0.24 \mathrm{deg} / \mathrm{s}$

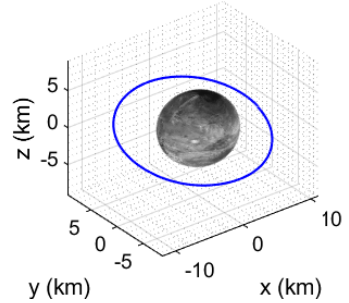

(a) Trajectory plot from GA results.

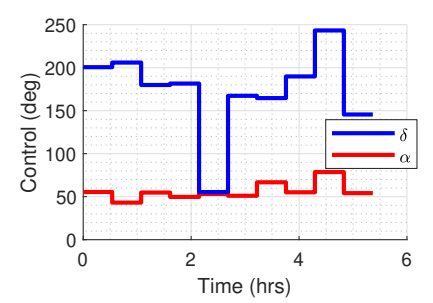

(b) Control history in clock $(\delta)$ and cone $(\alpha)$ angles

Fig. 6: Trajectory and control history for nominal orbit using GA with piece-wise constant interpolation.

However, although this solution brings the final state very close to the initial state, there is still a small error. Given the unstable nature of the orbit and its sensitivity to initial conditions, this means that a small error would quickly grow after a number of orbital revolutions. Fig. 7 shows how the nominal control law, applied over two orbits, causes the sail to diverge from the nominal trajectory. For this reason, the CTM method described in Section 5.2 is applied.

\subsection{Success Rates of CTM Method of Control Law Correction}

Applying the methods described in Section 5.2 , the Monte Carlo simulation will be performed for increasing radius $r_{0, \text { points }}$ of dispersion of the initial states. The success rate of each of the two parameters, outlined in Section 5.2.3. will then be measured for each $r_{0, \text { points }}$. Fig. 8 shows the success rates for $\epsilon_{\mathbf{u}_{\text {new }}}<$ 


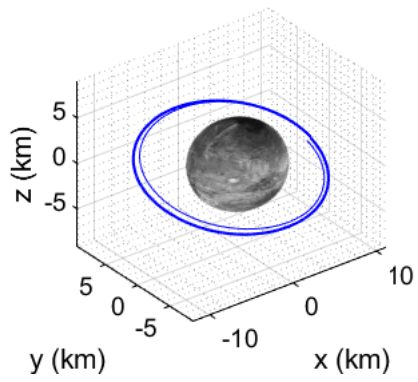

Fig. 7: Trajectory as in Fig. 6a propagated for two revolutions using same nominal control law shown in Fig. 6b

$|\delta \mathbf{s}|$ in red, and $\epsilon_{\mathbf{u}_{n e w}}<\epsilon_{\mathbf{u}_{\text {nominal }}}$ in blue up to $r_{0, \text { points }}=490 \mathrm{~m}$. Fig. 9 shows the success rates up to $r_{0, \text { points }}=9.8 \mathrm{~km}$.

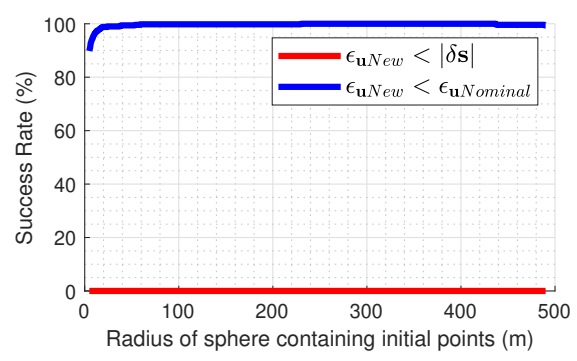

Fig. 8: Success rates of parameters $\epsilon_{\mathbf{u}_{n e w}}<|\delta \mathbf{s}|$ and $\epsilon_{\mathbf{u}_{\text {new }}}<\epsilon_{\mathbf{u}_{\text {nominal }}}$ with varying radius of sphere of dispersion of initial points.

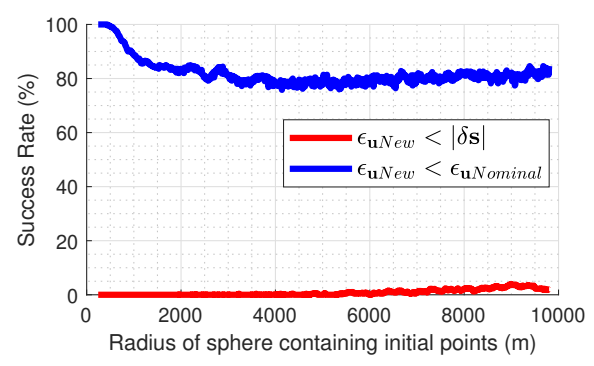

Fig. 9: Success rates of parameters $\epsilon_{\mathbf{u}_{n e w}}<|\delta \mathbf{s}|$ and $\epsilon_{\mathbf{u}_{n e w}}<\epsilon_{\mathbf{u}_{\text {nominal }}}$ with varying radius of sphere containing initial points.

For a sphere radius up to $500 \mathrm{~m}, \epsilon_{\mathbf{u}_{\text {new }}}<\epsilon_{\mathbf{u}_{\text {nominal }}}$ has very nearly $100 \%$ 
success, where $\epsilon_{\mathbf{u}_{\text {new }}}<|\delta \mathbf{s}|$ is never successful. As the radius of the sphere increases, there is a reduction in the success rate of $\epsilon_{\mathbf{u}_{\text {new }}}<\epsilon_{\mathbf{u}_{\text {nominal }}}$ until it settles around $80 \% . \epsilon_{\mathbf{u}_{\text {new }}}<|\delta \mathbf{s}|$ remains almost always unsuccessful until the sphere increases to a radius of around $4 \mathrm{~km}$, reaching a peak success rate of $4 \%$ at $r_{0, \text { points }} \approx 9 \mathrm{~km}$. However, given the linearisation of the system, the drop in success of $\epsilon_{\mathbf{u}_{\text {new }}}<\epsilon_{\mathbf{u}_{\text {nominal }}}$ may be an indication of the CTM being applied to trajectories which are not sufficiently close to the reference trajectory for the linearisation to be valid.

\subsection{Application of CTM to multiple revolutions}

In order to show the utility of using the CTM to update the control law, this section will present results where the CTM is used over multiple orbits to achieve periodicity.

Four test cases will be presented for different orbit inclinations. Each test case will show the target orbital elements, the orbital elements for the orbit obtained via the GA. The orbit selected from the GA is the one with the smallest difference between initial and final state. The orbital elements of the final periodic orbit to which the CTM method converges are also listed.

\subsubsection{Test Case 1}

The first test case is for an equatorial orbit with orbital elements shown in Table 4. The initial state for this test case, along with the controls and final time, form the solution vector of the Genetic Algorithm optimisation, as described in Section 5.1. The resulting orbit is taken as the nominal orbit, shown in red in all subsequent plots, and the CTM method is applied to update the control law on each subsequent orbit. Table 4 shows the orbital elements for the target orbit, the GA nominal orbit, and the final periodic orbit obtained using the CTM.

Fig. 10] shows the trajectory comparison of using the nominal control law for several subsequent orbital revolutions (Fig. 10a) and using the CTM method to update the control law for each orbit (Fig. 10b). As was made clear in Fig. 
8, the new control law, updated using the CTM, will almost always succeed in improving upon the error between the final state and the target final state than that which would have been achieved using the nominal control law.
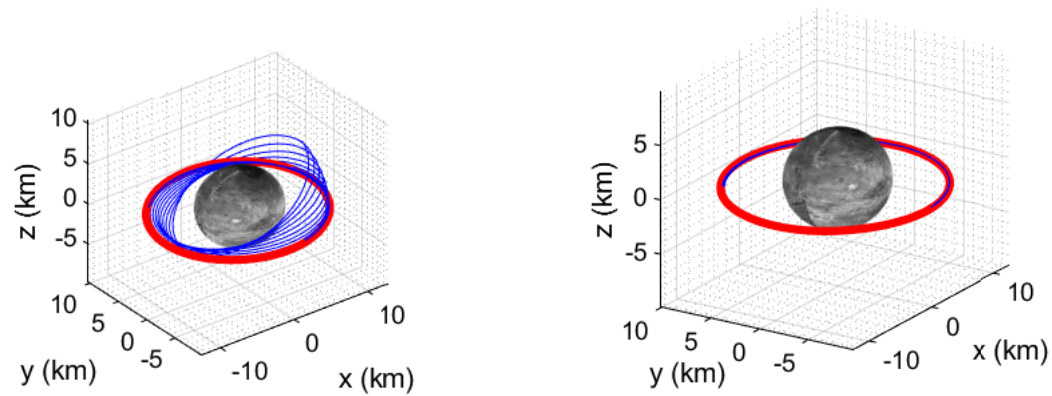

(a) Nominal orbit shown in red. Blue trajec- (b) Nominal orbit shown in red. Blue trajectory shows the divergent effect of continuing use tory represents 100 subsequent revolutions unof nominal control law for subsequent orbits, der control of the CTM method, where the conshown here for 10 revolutions. trol law is updated.

Fig. 10: Comparison of trajectories where the nominal control law is applied to subsequent orbital revolutions with the CTM employed to update the control law on each successive revolution.

Fig. 11 shows a comparison in the time history of the control law obtained from the GA alongside the control law obtained for the periodic orbit obtained through use of the CTM.

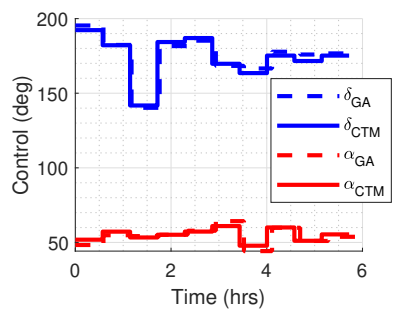

Fig. 11: Control law for the nominal orbit obtained from the GA (shown in broken lines) along with control law for the periodic orbit (shown in solid lines) for test case 1

In order to confirm the periodicity of the orbit obtained using the CTM method, Fig. 12 show the Poincaré sections in position (Fig. 12a) and velocity 
(Fig. 12b); it is clear that the trajectory converges to a periodic orbit and Table 5 shows that the orbital elements achieved through the CTM are very close to those of the nominal GA result.

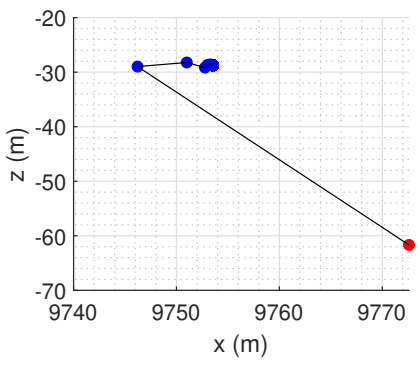

(a) Poincaré section in position.

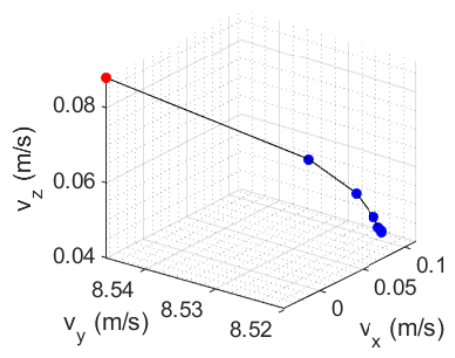

(b) Poincaré section in velocity.

Fig. 12: Poincaré sections in both position and velocity for 100 orbits where control has been updated using CTM method. Nominal orbit shown in red with all subsequent orbits in blue and the black connecting line showing the progression from the nominal to the final orbit.

Table 4: Principal orbital elements for the target orbit in test case 1. The nominal orbit is obtained from the GA and the final periodic orbit is obtained through the CTM control law updating.

\begin{tabular}{|c|c|c|c|}
\hline \hline & $a(\mathrm{~km})$ & $e$ & $i(\mathrm{deg})$ \\
\hline Target Orbit & 9.80 & 0.10 & 0 \\
\hline GA Result & 9.29 & 0.06 & 1.06 \\
\hline CTM Result & 9.12 & 0.07 & 0.32 \\
\hline \hline
\end{tabular}

\subsubsection{Test Case 2}

The second test case continues with the target orbit used in section 6.1. with orbital elements for the target orbit, the GA nominal orbit, and the final periodic orbit obtained via the CTM method shown in Table 5 .

Fig. 13 shows the trajectory comparison of using the nominal control law for any subsequent orbital revolution (Fig. 13a) and using the CTM method to update the control law for each orbit (Fig. 13b). 


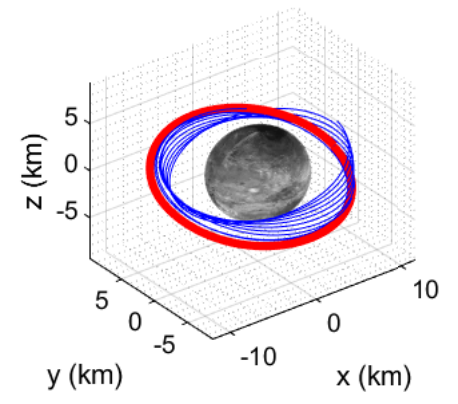

(a) Nominal orbit shown in red. Blue trajectory shows the divergent effect of continuing use of nominal control law for subsequent orbits, shown here for 10 orbits.

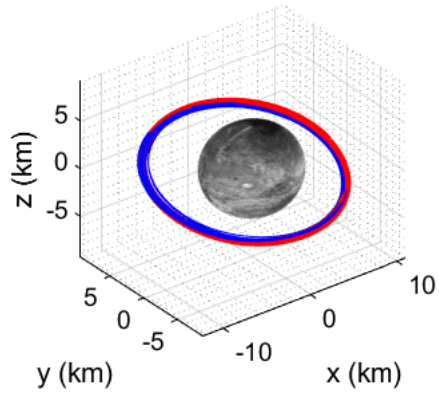

(b) Nominal orbit shown in red. Blue trajectory represents 100 subsequent orbits under control of the CTM method, where the control law is updated.

Fig. 13: Comparison of trajectories where the nominal control law is applied to subsequent orbits and where the CTM method is employed to update the control law on each successive orbit.

In order to confirm the periodicity of the orbit obtained updating the control through the CTM, Fig. 14 shows the Poincaré sections in position (Fig 14a) and velocity (Fig 14b). From these Poincaré sections, it is clear that the trajectory converges to a periodic orbit and Table 4 shows that the orbital elements achieved through the CTM are very close to those of the nominal GA result.

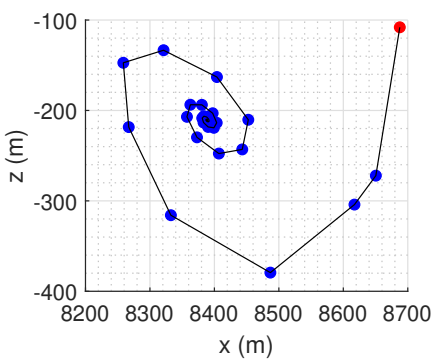

(a) Poincaré section in position.

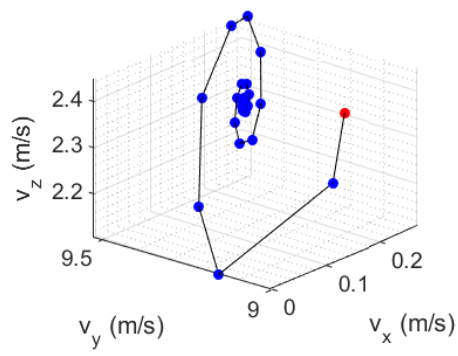

(b) Poincaré section in velocity.

Fig. 14: Poincaré sections in both position and velocity for 100 orbits where control has been updated using CTM method. Nominal orbit shown in red with all subsequent orbits in blue and the black connecting line showing the progression from the nominal to the final orbit. 
Table 5: Principal orbital elements for the target orbit in test case 2. The nominal orbit is obtained from the GA and the final periodic orbit is obtained by the CTM method of control law updating.

\begin{tabular}{|c|c|c|c|}
\hline \hline & $a(\mathrm{~km})$ & $e$ & $i(\mathrm{deg})$ \\
\hline Target Orbit & 9.80 & 0.10 & 15.00 \\
\hline GA Result & 8.84 & 0.01 & 14.54 \\
\hline CTM Result & 8.65 & 0.03 & 14.07 \\
\hline \hline
\end{tabular}

\subsubsection{Test Case 3}

In the third test case, the target orbit sits at an inclination of $30^{\circ}$, with the full target orbital elements shown in Table 6. Once again, there is an improvement in using the CTM method of updating the control law as compared to the nominal control law for subsequent orbits. Fig. 15 shows the trajectories for the nominal control over 10 orbits (Fig. 15a) and the trajectory where the CTM method is employed to update the control law (Fig. 15b) is shown for 100 orbital revolutions.

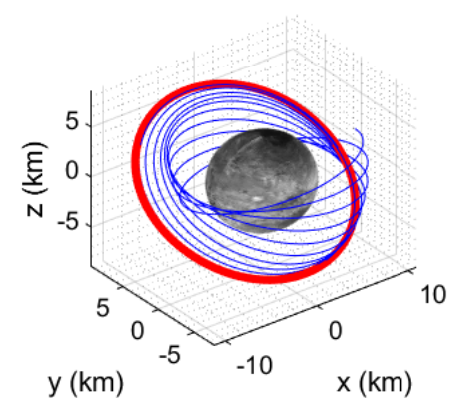

(a) Nominal orbit shown in red. Blue trajectory shows the divergent effect of continuing use of nominal control law for subsequent orbits, shown here for 10 orbital revolutions.

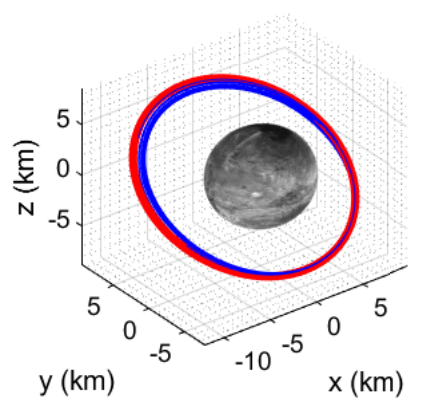

(b) Nominal orbit shown in red. Blue trajectory represents 100 subsequent orbital revolutions under updated control law through the CTM.

Fig. 15: Comparison of trajectories where the nominal control law is applied to subsequent orbits and where the CTM method is employed to update the control law on each successive orbital revolution. 
Once again, the method is successful and the periodicity of the final orbit is confirmed by the Poincaré sections shown in Fig. 16. The orbital elements for the final CTM orbit are shown alongside the GA result in Table6 6 and show close alignment.

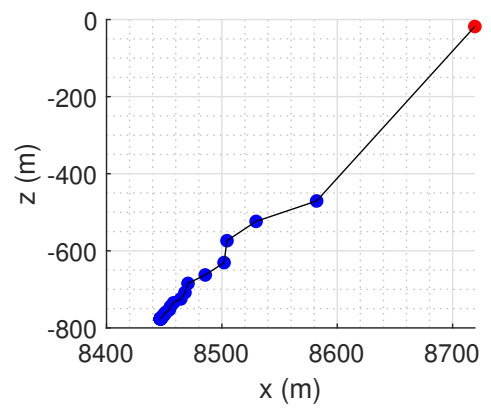

(a) Poincaré section in position.

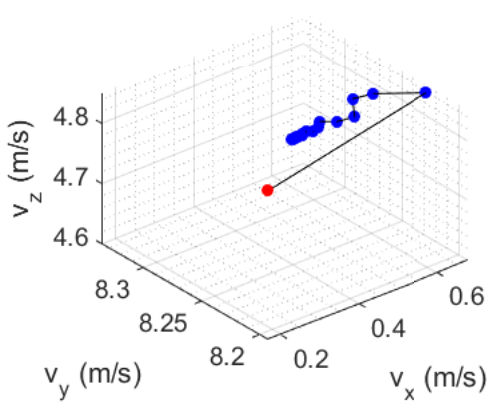

(b) Poincaré section in velocity.

Fig. 16: Poincaré sections in both position and velocity for 100 orbits where control has been updated using the CTM. Nominal orbit shown in red with all subsequent orbits in blue and the black line showing progression from nominal to final orbit.

Table 6: Principal orbital elements for the target orbit in test case 3. The nominal orbit is obtained from the GA and the final periodic orbit is obtained by the CTM method of control law updating.

\begin{tabular}{|c|c|c|c|}
\hline \hline & $a(\mathrm{~km})$ & $e$ & $i(\mathrm{deg})$ \\
\hline Target Orbit & 9.80 & 0.10 & 30.00 \\
\hline GA Result & 9.23 & 0.08 & 30.62 \\
\hline CTM Result & 8.65 & 0.03 & 29.77 \\
\hline \hline
\end{tabular}

\subsubsection{Test Case 4}

In the fourth test case, a higher inclination orbit is used. The initial Keplerian orbit parameters used for the GA, and those of the resulting orbit, are shown in Table 7 .

From Fig. 17, it would appear that at this inclination, there are no nearby periodic orbits controllable with the CTM method. This presents an interesting 
question of where this limit in inclination lies.

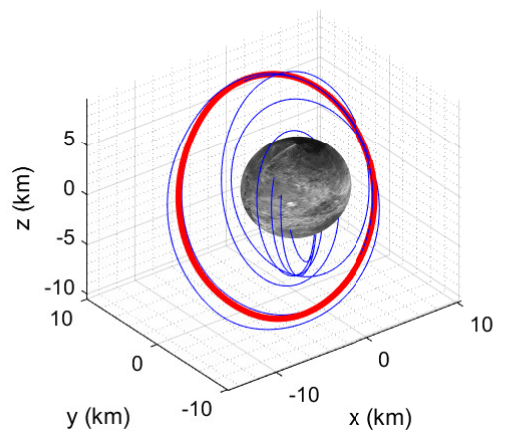

Fig. 17: CTM method applied over 4 orbital revolutions where GA result stems from initial orbit at $i=60^{\circ}$

Table 7: Principal orbital elements for the target orbit in test case 4. The nominal orbit is obtained from the GA and the final periodic orbit is obtained through the CTM control law updating.

\begin{tabular}{|c|c|c|c|}
\hline \hline & $a(\mathrm{~km})$ & $e$ & $i(\mathrm{deg})$ \\
\hline Target Orbit & 9.80 & 0.10 & 60.00 \\
\hline GA Result & 8.84 & 0.07 & 63.87 \\
\hline CTM Result & - & - & - \\
\hline \hline
\end{tabular}

\subsection{The Limiting Effect of Orbit Inclination}

Given the non-spherical shape of the body, it is expected that, as the right ascension of the ascending node, $\Omega$, is varied, there will be a corresponding change in the inclination limit for the CTM method. As such, and taking account of the symmetry of the ellipsoid, a study is conducted here for $\Omega \in[0,90]$ degrees. It is also expected that variation in other orbit parameters, semi-major axis $(a)$ and eccentricity $(e)$, will also have an effect. However, this section will make an initial analysis with $a=9.8 \mathrm{~km}$ and $e=0.10$.

Fig. 18 shows the boundary between the convergent and divergent regions for the CTM method. 


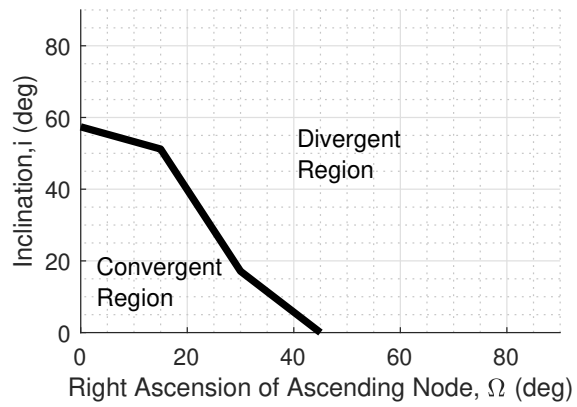

Fig. 18: Visualisation of the effect of changing $\Omega$ and $i$ on the convergence of the CTM method. The line represents the boundary at which point the CTM method cannot converge to a nearby periodic solution.

Further analysis is required to establish if variation of the other orbital parameters will improve the convergence of the method.

\section{Smoothing the Control Law for Realistic Application}

Up to this point, the control laws have been interpolated using a piece-wise constant method. However, this method required instantaneous changes in sail attitude. As discussed in Section 5, this is not possible due to limited attitude actuation capacity of the sail. Therefore, in order to present a realistic solution, the control interpolation method is changed to cubic splines, and the process is restarted. The resulting GA trajectory and control law for Test Case 2 (section 6.3.2 are shown in Fig. 19

The magnitude of the rigid-body angular velocity, $\dot{\gamma}$, and angular accelerations, $\ddot{\gamma}$, required for this nominal control are shown in Fig. 20. The angle between two attitudes is given by (Peloni et al. 2016):

$$
\cos (\gamma)=\hat{\mathbf{n}}\left(t_{i}\right) \cdot \hat{\mathbf{n}}\left(t_{i+1}\right)
$$

where $\hat{\mathbf{n}}$ is the unit vector of the sail normal, $t$ is the time and $i$ is the indexing variable. As such, the angular velocity, $\dot{\gamma}$, is the time rate of change of this angle. Likewise, the angular acceleration, $\ddot{\gamma}$, is the time rate of change of $\dot{\gamma}$. 


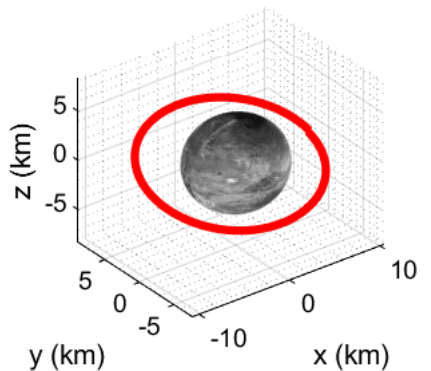

(a) Nominal trajectory from GA using cubic spline interpolated control law

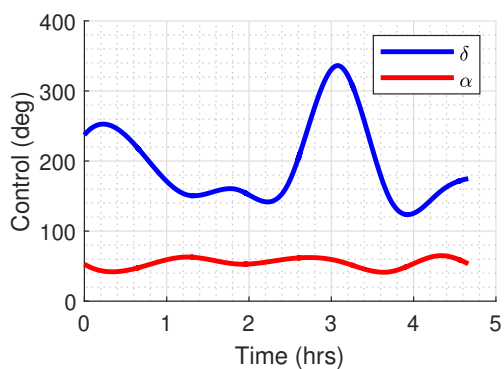

(b) Nominal control law using cubic spline interpolation

Fig. 19: Nominal trajectory and control law using cubic spline interpolation on control in the GA

For the nominal orbit, the optimal values of the objectives from the GA are given in Table 9

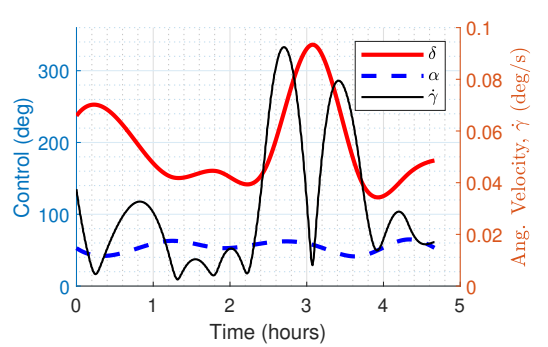

(a) Magnitude of rigid-body angular velocity, $\dot{\gamma}$

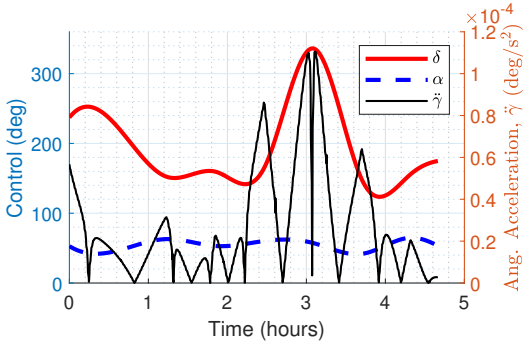

(b) Angular acceleration, $\ddot{\gamma}$

Fig. 20: Rigid-body angular velocity, $\dot{\gamma}$, and angular accelerations, $\ddot{\gamma}$, plotted against control history for nominal orbit

With the nominal trajectory and control established, the CTM method is once again used to update the control law to ensure a periodic solution. As before, the simulation is performed for 100 subsequent orbital revolutions. Fig. 21 shows the nominal trajectory in red with the 100 subsequent orbits in blue.

Fig. 22 shows the Poincaré sections in position and velocity where, once again, the nominal orbit is in red and all subsequent orbits in blue. The black line highlights the sequence from nominal to periodic. Finally, Fig. 23 shows the 
comparison of the GA control law (dashed line) with the CTM control law (solid line) for the final periodic orbit.

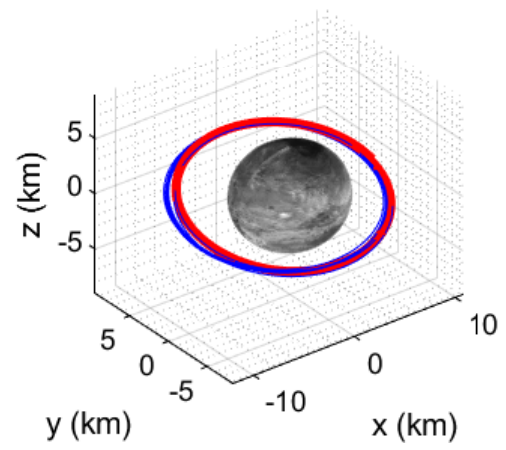

Fig. 21: Trajectory plot of nominal orbit with spline-interpolated control (red), and subsequent propagation for 100 orbital revolutions with the control law updated at each orbit using the CTM.

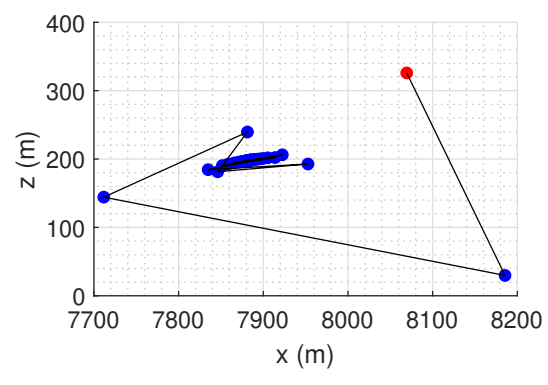

(a) Poincaré section in position

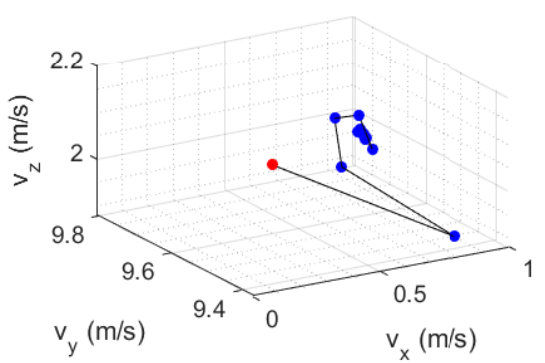

(b) Poincaré section in velocity

Fig. 22: Poincaré sections in both position and velocity for 100 orbital revolutions where control has been interpolated using cubic splines and updated using the CTM. Nominal orbit shown in red with all subsequent orbits in blue and the black line showing progression from nominal to final orbit.

As in Sec. 6.3.1, the results show that the CTM method has converged to a stable periodic solution. As the control law has been interpolated using cubic splines, a smooth transition in sail orientation is obtained, allowing for the slower rotational rates of a realistic sail. Table 8 shows the orbital elements of the targeted orbit, the nominal orbit resulting from the GA and the periodic 


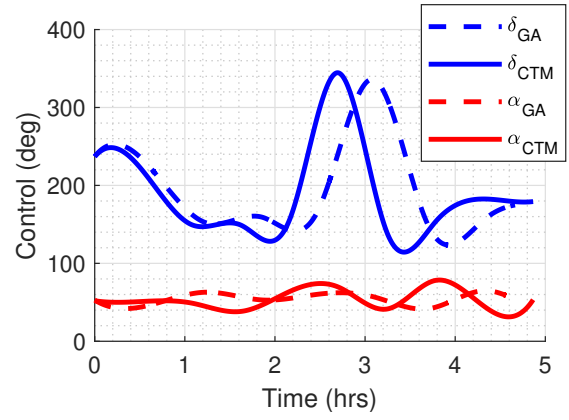

Fig. 23: Comparison of the control law obtained from the Genetic Algorithm (GA) and the Control Transition Matrix (CTM) periodic solution.

orbit obtained through the CTM.

Table 8: Principal orbital elements for target orbit, GA nominal orbit and CTM periodic orbit, using cubic spline interpolation.

\begin{tabular}{|c|c|c|c|}
\hline \hline & $a(\mathrm{~km})$ & $e$ & $i(\mathrm{deg})$ \\
\hline Target Orbit & 9.80 & 0.10 & 15.00 \\
\hline GA Result & 8.27 & 0.03 & 12.89 \\
\hline CTM Result & 8.03 & 0.09 & 11.73 \\
\hline \hline
\end{tabular}

The magnitude of the rigid-body angular velocity and the angular acceleration are plotted with the control history for the CTM-updated periodic orbit in Fig. 24. Table 9 shows the values of each objective for both the nominal orbit from the GA, and the CTM-updated periodic orbit.

Table 9: Objective function values for the GA nominal orbit and CTM obtained periodic orbit, where cubic splines interpolation method is used.

\begin{tabular}{|c|c|c|}
\hline \hline & $\left\|\mathbf{s}\left(t_{f}\right)-\mathbf{s}\left(t_{0}\right)\right\|$ & $\Delta \dot{\gamma}(\mathrm{deg} / \mathrm{s})$ \\
\hline GA Nominal Orbit & 0.04 & 0.46 \\
\hline CTM Periodic Orbit & 0.01 & 0.63 \\
\hline
\end{tabular}




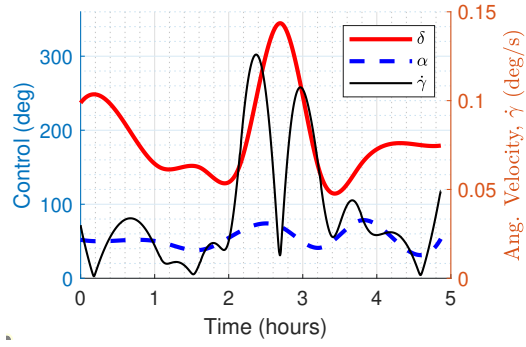

(a) Magnitude of rigid-body angular velocity

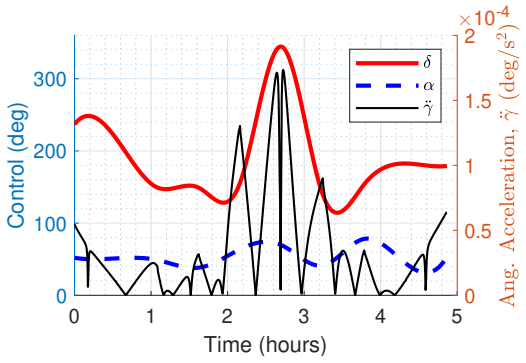

(b) Angular Acceleration

Fig. 24: Rigid-body angular velocity and angular accelerations plotted against control history for CTM periodic orbit

\subsection{Torque Analysis}

In order to further understand the implication on a realistic sail, this section will calculate the torques required for attitude tracking. The sails will be considered as square flat plates and the spacecraft bus is considered to be a cube. Fig. 25 shows the principal axes of inertia for a plate with negligible thickness.

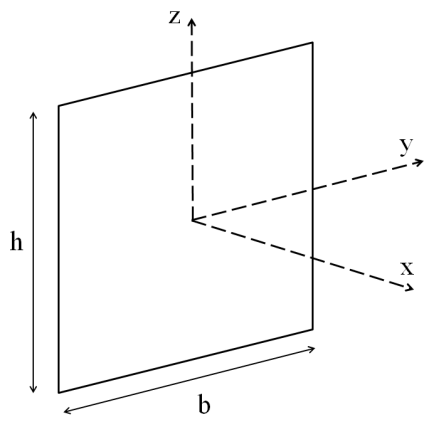

Fig. 25: Principal axes of inertia for a very thin square plate

In order to take the most conservative estimate of torque requirements, the analysis will be conducted around one of these principal axes, where the moments of inertia are highest. The moment of inertia (MOI) for a square flat plate $(b=h)$ are highest around the $x$-axis. However, rotations around the $x$-axis, shown in Fig. 25, are irrelevant for the sail acceleration, and hence not considered. Therefore, rotations in this analysis will be conducted around the 
$z$-axis of Fig. 25. As such, and with $b=h$, the moment of inertia for a square flat plate around the principal $z$-axis are given by (Meriam and Kraige, 1998):

$$
I_{z z}=\frac{1}{12} m_{p} h_{p}^{2}
$$

and for a cube, around the same axis, the MOI is given by:

$$
I_{z z}=\frac{1}{6} m_{c} h_{c}^{2}
$$

where $m_{p}$ is the mass of the plate, $m_{c}$ is the mass of the cube, $h_{p}$ is the edge length of the plate and $h_{c}$ is the edge length of the cube. The centroid of the sail and spacecraft bus will be considered coincident and their principal axes aligned, so the total MOI of the spacecraft is the sum of the bus' and the sail's. The torque can then be calculated from the angular accelerations, $\ddot{\gamma}$ which are shown in Figs 20 and 24 for the Nominal and CTM orbits respectively. The torque is given by:

$$
\tau=I \ddot{\gamma}
$$

Three classes of spacecraft, of different size and mass, will be compared. Throughout each, the characteristic acceleration of $a_{c}=0.2 \mathrm{~mm} / \mathrm{s}^{2}$ is maintained. The details of each sail and spacecraft bus are listed in Table 10. In order to establish the mass of the sail assembly, Ceriotti et al. (2012) state that for a near-term sail, an areal density for the sail assembly of $10 \mathrm{~g} / \mathrm{m}^{2}$ should be used. To establish the correct dimensions of the spacecraft bus, a cube density of $142.4 \mathrm{~kg} / \mathrm{m}^{3}$ is maintained. This figure was obtained after calculating the spaceraft bus mass for the $2 \mathrm{~m}$ edge length sailcraft with characteristic acceleration of $\mathrm{a}_{c}=0.2 \mathrm{~mm} / \mathrm{s}^{2}$ and then calculating the density for such a mass in a $(10 \mathrm{~cm})^{3}$ volume.

The orbit from Test Case 2 (Secs. 6.3.2 and 7 ) is again used here to demonstrate the required torques to achieve the desired control. First, the results for the nominal orbit are shown in Fig. 26 for the three different sizes of sail. Fig. 27 shows the required torques for the CTM controlled orbit. 
Table 10: Sail and spacecraft bus dimensions, mass and moments of inertia

\begin{tabular}{|c|c|c|c|}
\hline \hline Sail Edge Length $(\mathrm{m})$ & 2 & 10 & 20 \\
Sail Area $\left(\mathrm{m}^{2}\right)$ & 4.00 & 100.00 & 400.00 \\
Sail Assembly Mass $(\mathrm{kg})$ & 0.10 & 1.00 & 4.00 \\
Sail MOI $\left(\mathrm{kg} \cdot \mathrm{m}^{2}\right)$ & 0.01 & 8.33 & 133.33 \\
Spacecraft Bus Edge Length $(\mathrm{m})$ & 0.1 & 0.29 & 0.46 \\
Spacecraft Bus Mass $(\mathrm{kg})$ & 0.14 & 3.56 & 14.24 \\
Spacecraft Bus MOI $\left(\mathrm{kg} \cdot \mathrm{m}^{2}\right)$ & $2.37 \times 10^{-4}$ & 0.05 & 0.51 \\
\hline \hline
\end{tabular}

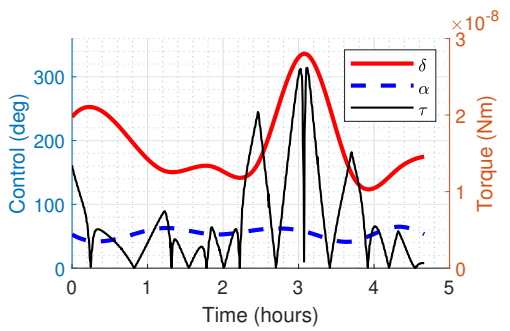

(a) Sail with edge length of $2 \mathrm{~m}$

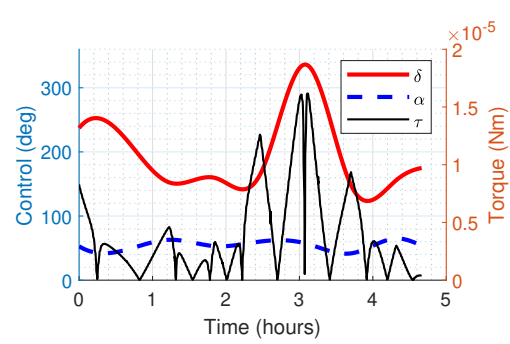

(b) Sail with edge length of $10 \mathrm{~m}$

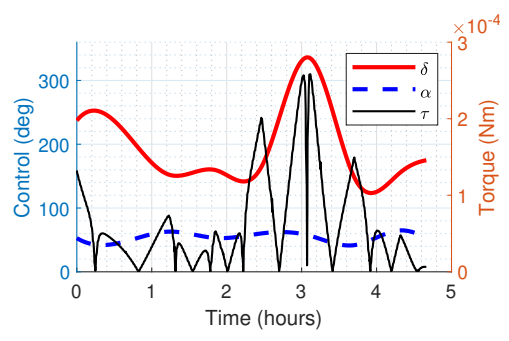

(c) Sail with edge length of $20 \mathrm{~m}$

Fig. 26: Nominal orbit torque, $\tau$, shown against control history for square sail

The maximum torques for each case shown in Figs. 26 and 27 are shown in Table 11 To provide some context, Plante et al. (2017) discuss the ACDS system design for The Planetary Society's LightSail 2 mission. In their study of changes in inclination, the maximum torques in yaw, which are provided by a reaction wheel, are given as $\tau=0.06 \mathrm{Nm}$. In their work on a Sun pointing solar sail ACDS system design in Earth orbit, Wie (2004) calculates that the 


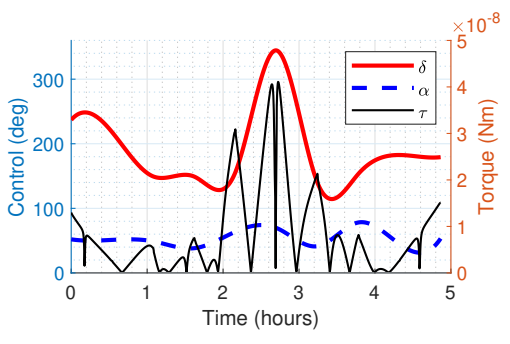

(a) Sail with edge length of $2 \mathrm{~m}$

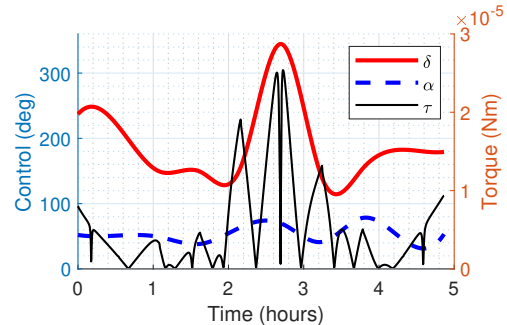

(b) Sail with edge length of $10 \mathrm{~m}$

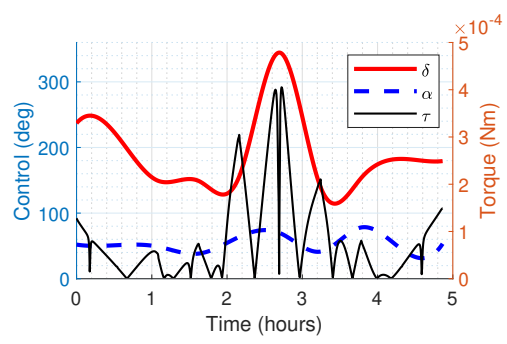

(c) Sail with edge length of $20 \mathrm{~m}$

Fig. 27: Attitude torque $\tau$ on CTM-controlled orbit, shown against control history for square sails with side lengths of $2 \mathrm{~m}, 10 \mathrm{~m}$ and $20 \mathrm{~m}$.

maximum required control torques will have a magnitude of $\tau \approx 0.002 \mathrm{Nm}$. Therefore, the torques required in this work would appear feasible for a realistic sail in the context of previous work in the literature.

Table 11: Maximum Torque Values

\begin{tabular}{|c|c|c|c|}
\hline \hline Sail Edge Length $(\mathrm{m})$ & 2 & 10 & 20 \\
\hline Nominal Orbit $(\mathrm{Nm})$ & $2.62 \times 10^{-8}$ & $1.62 \times 10^{-5}$ & $2.58 \times 10^{-4}$ \\
CTM Orbit $(\mathrm{Nm})$ & $4.11 \times 10^{-8}$ & $2.54 \times 10^{-5}$ & $4.05 \times 10^{-4}$ \\
\hline \hline
\end{tabular}

\section{Conclusions}

In this work, a method of mitigating the effects of perturbations due to the non-spherical shape of a body using a solar sail have been presented. Given the system sensitivities, it is desirable to select an optimisation method which requires no initial guess of the solution from the user. This work uses a Genetic 
Algorithm to conduct a multi-objective optimisation to obtain a periodic orbit while also minimising the effort required by the sail to do so. As this method required no initial input from the user, other than to define the optimisation parameters and their limits, it allows for a far more robust way in obtaining the optimal solution.

This method proved successful in establishing near-periodic orbits while also presenting solutions with low effort values. It was found that, although the orbits were very nearly periodic, the small discrepancy between final and initial state meant that the control law was not valid for any subsequent orbits. As such, a method of updating the control law for any subsequent orbit was presented. This method used the Control Transition Matrix (CTM), a matrix containing information on the effects of variation in control on final state errors, to update the control law, allowing for stable periodic orbits to be established.

This method of updating the control law was found to be limited by the orbital inclination. This inclination limit was also found to vary with the right ascension of the orbit's ascending node. It is found that the magnitude of the tesseral and sectoral harmonics is small relative to that of the zonal harmonics for this body. As the zonal harmonics dominate, and are invariant in longitude, the asteroid rotation has negligible effect on analysing the effect of parameters which are longitudinal, such as varying $\Omega$. Analysis on this variation was presented with a boundary between the convergent and divergent regions. It is expected that other orbital parameters (such as semi-major axis and eccentricity) will also affect this convergence study. However, this work presents an initial analysis where these parameters remain fixed.

Finally, to generate a realistic, smooth control law, the piece-wise-continuous interpolation of the control was replaced with cubic splines, which allowed gradual transitions in attitude. The CTM method was once again successful in establishing a stable periodic orbit from this updated nominal orbit, with a small increase in required effort from the sail. Analysis of the torque which would be required from the ACDS system were then shown. These results showed that the maximum required torques lay well within the maximum torques of realistic 
sail examples from the literature.

\section{Acknowledgements}

Iain Moore would like to thank the James Watt School of Engineering (University of Glasgow) and the Engineering and Physical Sciences Research Council (EPSRC) for supporting this work.

Matteo Ceriotti would like to thank the James Watt School of Engineering (University of Glasgow) and the Institution of Mechanical Engineers (IMechE Conference Grant EAC/KDF/OFFER/19/046) for supporting this work.

The authors thank Colin McInnes, Merel Vergaaij, Alessandro Peloni and Giulia Viavattene for their expertise and advice.

\section{References}

Bando, M., Yamakawa, H., 2011. Near-earth asteroid flyby survey mission using solar sailing technology. The Journal of the Astronautical Sciences 58, 569581. doi $10.1007 / \mathrm{BF} 03321532$.

Biggs, J.D., McInnes, C.R., 2009. Time-delayed feedback control in astrodynamics. Journal of Guidance, Control, and Dynamics 32, 1804-1820. doi:10.2514/1.43672.

Ceriotti, M., Diedrich, B.L., McInnes, C.R., 2012. Novel mission concepts for polar coverage: An overview of recent developments and possible future applications. Celestial Mechanics and Dynamical Astronomy 114, 151-180. doi:10.1007/s10569-012-9422-2.

Ceriotti, M., Sanchez, J.P., 2016. Control of asteroid retrieval trajectories to libration point orbits. Acta Astronautica 126, 342-353. doi 10.1016/ j.actaastro.2016.03.037.

Coverstone-Carroll, V., Hartmann, J.W., Mason, W.J., 2000. Optimal multiobjective low-thrust spacecraft trajectories. Computer Methods in Applied 
Mechanics and Engineering 186, 387-402. doi 10.1016/S0045-7825(99) 00393-X

Cox, B., Cohen, A., 2017. Forces of nature. Harper Collins Publishing, London, UK.

Dachwald, B., Boehnhardt, H., Broj, U., Geppert, U.R., Grundmann, J.T., Seboldt, W., Seefeldt, P., Spietz, P., Johnson, L., Kührt, E., et al., 2014. Gossamer roadmap technology reference study for a multiple NEO rendezvous mission, in: Advances in Solar Sailing. Springer, pp. 211-226. doi10.1007/ 978-3-642-34907-2_15.

Dirkx, D., Mooij, E., 2017. Conceptual shape optimization of entry vehicles: Applied to capsules and winged fuselage vehicles. Springer Aerospace Technology, Cham, Switzerland. URL: 10.1007/978-3-319-46055-0, doi 10.1007/ 978-3-319-46055-0.

Farres, A., Ceriotti, M., 2012. Solar sail station keeping of high-amplitude vertical lyapunov orbits in sun-earth system, in: 63rd International Astronautical Congress (IAC 2012), Naples, Italy.

Farrés, A., Jorba, A., 2011. On the station keeping of a solar sail in the elliptic sun-earth system. Advances in Space Research 48, 1785-1796. doi 10.1016/ j.asr.2011.02.004.

Farrés, A., Jorba, A., 2014. Station keeping of a solar sail around a halo orbit. Acta Astronautica 94, 527-539. doi:10.1016/j.actaastro.2012.07.002.

Farrés, A., Jorba, À., 2016. Station Keeping Strategies for a Solar Sail in the Solar System. Springer International Publishing, Cham. pp. 83115. URL: https://doi.org/10.1007/978-3-319-27464-5_3, doi 10 . 1007/978-3-319-27464-5_3.

Farrés, A., Jorba, , 2012. A dynamical system approach for the station keeping of a solar sail. The Journal of the Astronautical Sciences 56, 199-230. doi 10. $1007 /$ bf 03256549 
Gozdziewski, Krzysztof; Maciejewski, A.J., 1998. Nonlinear stability of the lagrangian libration points in the chermnykh problem. Celestial Mechanics and Dynamical Astronomy 70, 41-58. doi:10.1023/A:1008250207046

Grundmann, J.T., Bauer, W., Biele, J., Boden, R., Ceriotti, M., Cordero, F., Dachwald, B., Dumont, E., Grimm, C.D., Herčík, D., et al., 2019. Capabilities of gossamer-1 derived small spacecraft solar sails carrying mascot-derived nanolanders for in-situ surveying of NEAs. Acta Astronautica 156, 330-362. doi $10.1016 / \mathrm{j}$. actaastro.2018.03.019.

Grundmann, J.T., Bauer, W., Biele, J., Cordero, F., Dachwald, B., Koncz, A., Krause, C., Mikschl, T., Montenegro, S., Quantius, D., et al., 2015. From sail to soil-getting sailcraft out of the harbour on a visit to one of earths nearest neighbours, in: 4th IAA Planetary Defense Conference-PDC 2015.

Guibout, V., Scheeres, D.J., 2003. Stability of surface motion on a rotating ellipsoid. Celestial Mechanics and Dynamical Astronomy 87, 263-290. doi 10. 1023/B:CELE.0000005720.09027.ee.

Hesar, S.G., Scheeres, D.J., McMahon, J.W., 2016. Sensitivity analysis of the osiris-rex terminator orbits to maneuver errors. Journal of Guidance, Control, and Dynamics , 81-95, doi $10.2514 / 1 . G 002058$.

Ho, T.M., Baturkin, V., Grimm, C., Grundmann, J.T., Hobbie, C., Ksenik, E., Lange, C., Sasaki, K., Schlotterer, M., Talapina, M., et al., 2017. Mascot-the mobile asteroid surface scout onboard the hayabusa 2 mission. Space Science Reviews 208, 339-374. doi:10.1007/s11214-016-0251-6.

Hu, X., Jekeli, C., 2015. A numerical comparison of spherical, spheroidal and ellipsoidal harmonic gravitational field models for small non-spherical bodies. Geodesy and Geodynamics 89, 159-177. doi 10.1007/s00190-014-0769-x.

Hughes, G., McInnes, C.R., 2004. Small-body encounters using solar sail propulsion. Journal of spacecraft and rockets 41, 140-150. doi 10.2514/1.9277. 
Macdonald, M., McInnes, C., 2005. Realistic earth escape strategies for solar sailing. Journal of Guidance, Control, and Dynamics 28, 315-323. doi 10 . $2514 / 1.5165$.

McInnes, C.R., 1999. Solar sailing: Technology, dynamics and mission applications. Springer Praxis, Chichester, UK.

McNutt, L., Johnson, L., Kahn, P., Castillo-Rogez, J., Frick, A., 2014. Nearearth asteroid (NEA) scout, in: AIAA Space 2014 Conference and Exposition, p. 4435 . doi $10.2514 / 6.2014-4435$.

Meriam, J., Kraige, L., 1998. Engineering Mechanics: Dynamics. Fourth ed., John Wiley \& Sons Inc, New York.

Mota, M.L., Rocco, E.M., 2019. Equilibrium points stability analysis for the asteroid 21 lutetia. Journal of Physics: Conference Series 1365. doi 10.1088/ $1742-6596 / 1365 / 1 / 012007$

Oliveira, T.C., Prado, A.F.B.A., 2014. Evaluating orbits with potential to use solar sail for station-keeping maneuvers, in: 2nd IAA Conference on Dynamics and Control of Space Systems (DYCOSS), International Academy of Astronautics.

Peloni, A., Ceriotti, M., Dachwald, B., 2016. Solar sail trajectory design for a multiple near-earth asteroid rendezvous mission. Journal of Guidance, Control, and Dynamics 39, 2712-2724. doi 10.2514/1.G000470

Plante, B.A., Spencer, D.A., Betts, B., Chait, S., Bellardo, J.M., Diaz, A., Pham, I., 2017. LightSail 2 ADCS: From simulation to mission readiness, in: Proceedings of the 4th International Symposium on Solar Sailing (ISSS 2017), Kyoto, Japan.

Pravec, P., Harris, A., Michalowski, T., 2002. Asteroid rotations. University of Arizona Press, Tuscon, Arizona. pp. 113-122. 
Rao, A.V., Benson, D.A., Darby, C., Patterson, M.A., Francolin, C., Sanders, I., Huntington, G.T., 2010. Algorithm 902: GPOPS, a MATLAB software for solving multiple-phase optimal control problems using the Gauss pseudospectral method. ACM Transactions on Mathematical Software 37, 22-39. doi:10.1145/1731022.1731032

Rocco, E.M., 2019. Evaluation of the approaching trajectories for landing on the asteroid 216 Kleopatra, in: Proceedings of the XL Ibero-Latin-American Congress on Computational Methods in Engineering.

Rocco, E.M., Souza, M., Prado, A., 2003. Multi-objective optimization applied to satellite constellations i: Formulation of the smallest loss criterion, in: Proceedings of the 54st International Astronautical Congress (IAC'03), Bremen, Germany. doi $10.2514 / 6$. IAC-03-A.3.01.

Rocco, E.M., de Oliveira e Souza, M.L., de Almeida Prado, A.F.B., 2013. Station keeping of constellations using multiobjective strategies. Mathematical Problems in Engineering 2013. doi:10.1155/2013/476451.

Rossi, A; Marzari, F.F.P., 1999. Orbital evolution around irregular bodies. Earth Planets Space 51, 1173-1180. doi 10.1186/BF03351592.

Sarli, B.V., Tsuda, Y., 2017. Hayabusa 2 extension plan: Asteroid selection and trajectory design. Acta Astronautica 138, 225-232. doi $10.1016 / \mathrm{j}$. actaastro.2017.05.016

Scheeres, D.J., 1993. Satellite dynamics about tri-axial ellipsoids. Technical Report. NASA Jet Propulsion Laboratory.

Scheeres, D.J., 1994. Dynamics about uniformly rotating triaxial ellipsoids: Applications to asteroids. Icarus 110, 225-238. doi:10.1006/icar.1994. 1118

Scheeres, D.J., 1999. The effect of $C_{22}$ on orbit energy and angular momentum. Celestial Mechanics and Dynamical Astronomy 73, 339-348. doi 10.1023/A: 1008384021964 
Scheeres, D.J., 2004. Close proximity operations for implementing mitigation strategies, in: Planetary Defense Conference: Protecting Earth from Asteroids. doi $10.2514 / 6.2004-1445$.

Scheeres, D.J., 2011. Orbital motion in strongly perturbed environments: applications to asteroid, comet and planetary satellite orbiters. Springer-Verlag, Berlin, Heidelberg.

Scheeres, D.J., 2012. Orbital mechanics about small bodies. Acta Astronautica 72, 1-14. doi $10.1016 / \mathrm{j}$.actaastro.2011.10.021

Scheeres, D.J., Ostro, S.J., Hudson, R.S., Werner, R.A., 1996. Orbits close to asteroid 4769 castalia. Icarus 121, 67-87. doi 10.1006/icar.1996.0072

Sebera, J., Bezdek, A., Henych, I.P.T., 2016. Spheroidal models of the exterior gravitational field of asteroids Bennu and Castalia. Icarus 272, 70-79. doi:10. $1016 / \mathrm{j}$. icarus.2016.02.038

Takahashi, Y., Scheeres, D.J., 2014. Small body surface gravity fields via spherical harmonic expansions. Celestial Mechanics and Dynamical Astronomy 119, 169-206. doi $10.1007 /$ s10569-014-9552-9.

Vallado, D.A., 2013. Fundementals of astrodynamics and applications. Microcosm Press, Hawthorne, California.

Venditti, F.C.F., Prado, A.F.B.A., 2015. Mapping orbits regarding perturbations due to the gravitational field of a cube. Mathematical Problems in Engineering 2015, 1-11. doi $10.1155 / 2015 / 493903$.

Viale, A., McInnes, C., Ceriotti, M., 2019. Dynamics of a non-rigid orbital siphon at a near-Earth asteroid, in: 2019 AAS/AIAA Astrodynamics Specialist Conference.

Werner, R.A., 1994. The gravitational potential of a homogeneous polyhedron or don’t cut corners. Celestial Mechanics and Dynamical Astronomy 59, 253278. doi $10.1007 / \mathrm{BF} 00692875$. 
Werner, R.A., Scheeres, D.J., 1997. Exterior gravitation of a polyhedron derived and compared with harmonic and mascon gravitation representations of asteroid 4769 Castalia. Celestial Mechanics and Dynamical Astronomy 65, 313-344. doi $10.1007 / \mathrm{BF} 00053511$.

Wie, B., 2004. Solar sail attitude control and dynamics, part 1. Journal of Guidance, Control, and Dynamics 27, 526-535. doi 10.2514/1.11134.

Wiesel, W.E., 2010. Modern Astrodynamics. Second ed., Aphelion, Beavercreek, Ohio.

Yoshikawa, M., Fujiwara, A., Kawaguchi, J., 2006. Hayabusa and its adventure around the tiny asteroid itokawa. Proceedings of the International Astronomical Union 2, 323-324. doi $10.1017 /$ S174392130701085X.

Zeng, X., Gong, S., Li, J., 2014. Fast solar sail rendezvous mission to near earth asteroids. Acta Astronautica 105, 40-56. doi $10.1016 / \mathrm{j}$.actaastro. 2014. 08.023 . 